\title{
A new era of personalized medicine for cystic fibrosis - at last!
}

\author{
Bradley S Quon MD MSc FRCPC ${ }^{1,2}$, Pearce G Wilcox MD FRCPC ${ }^{1,2}$
}

BS Quon, PG Wilcox. A new era of personalized medicine for cystic fibrosis - at last! Can Respir J 2015;22(5):257-260.

The gene responsible for cystic fibrosis (CF) was discovered 25 years ago. This breakthrough has enabled a sophisticated understanding of how various mutations lead to specific alterations in the structure and function of the CF transmembrane regulator (CFTR) protein. Until recently, all therapies in CF were focused on ameliorating the downstream consequences of CFTR dysfunction. High-throughput drug screening approaches have yielded compounds that can modify CFTR structure and function, thus targeting the basic defect in CF. The present article describes the CFTR mutational classes, reviews mutation-specific therapies currently in latephase clinical development, and highlights research opportunities and challenges with personalized medicine in CF.

Key Words: CFTR modulators; CFTR mutation; Cystic fibrosis; Personalized medicine

$\mathrm{R}$ ecently, the cystic fibrosis (CF) community celebrated the 25th Ranniversary of the discovery of the gene responsible for CF (1). While many believed a cure for CF would result from gene therapy, this promise has yet to be realized due to significant challenges in the implementation of this therapeutic strategy, as detailed in a recent review (2). However, the discovery has enabled a sophisticated understanding of how various mutations in the gene encoding the CF transmembrane conductance regulator (CFTR) lead to alterations in the structure and function of the resulting protein. High-throughput small molecule screening programs have yielded compounds that modulate CFTR function. The present focused review describes the CFTR mutational classes, reviews mutation-specific therapies currently in late-phase clinical development, and highlights research opportunities and challenges with personalized medicine in CF.

\section{CFTR MUTATIONAL CLASSES}

To understand personalized treatments designed to correct the basic CFTR defect, a working understanding of the various mutational classes and the mechanisms of CFTR dysfunction are required. Nearly 2000 mutations have been reported in the CFTR gene (3); however, to date, only 127 are known to be disease causing (4). Of these mutations, only 15 occur with an overall frequency $>1 \%$ in Canada, but there is variation in the regional prevalence of specific mutations across Canada.

Six mutational classes of CFTR dysfunction have been described based on their impact on CFTR expression, trafficking, stability and function (Table 1). Class I mutations are most commonly nonsense mutations that cause premature stop codons and, hence, no functional CFTR protein. Translational readthrough agents are small molecules that induce selective ribosomal readthrough of messenger RNA containing a premature stop codon, allowing translation of the full-length protein (Figure 1). Class II mutations are missense mutations that cause amino acid deletions that result in protein misfolding and failure of the CFTR protein to be transported to the cell surface. CFTR correctors are small molecules designed to correct cellular misprocessing of CFTR (eg, folding) to facilitate trafficking from the endoplasmic reticulum to the cell surface (Figure 1). Class III mutations are also missense mutations but cause amino acid substitutions that result in

\section{Enfin! Une nouvelle ère de médecine personnalisée pour la fibrose kystique}

Le gène responsable de la fibrose kystique (FK) a été découvert il y a 25 ans. Grâce à cette percée, on comprend mieux les modifications précises à la structure et à la fonction de la protéine du régulateur de conductance transmembranaire de la FK (CFTR), responsables des diverses mutations. Jusqu'à récemment, tous les traitements de la FK étaient axés sur l'atténuation des conséquences en aval de la dysfonction de la protéine CFTR. Le criblage à haut rendement a permis de créer des composés qui peuvent modifier la structure et la fonction CFTR, ciblant ainsi la défectuosité fondamentale de la FK. Le présent article décrit les classes de mutation du gène CFTR, aborde les traitements propres aux mutations qui en sont aux dernières phases de développement clinique et fait ressortir les possibilités de recherche et les problèmes liés à la médecine personnalisée de la FK.

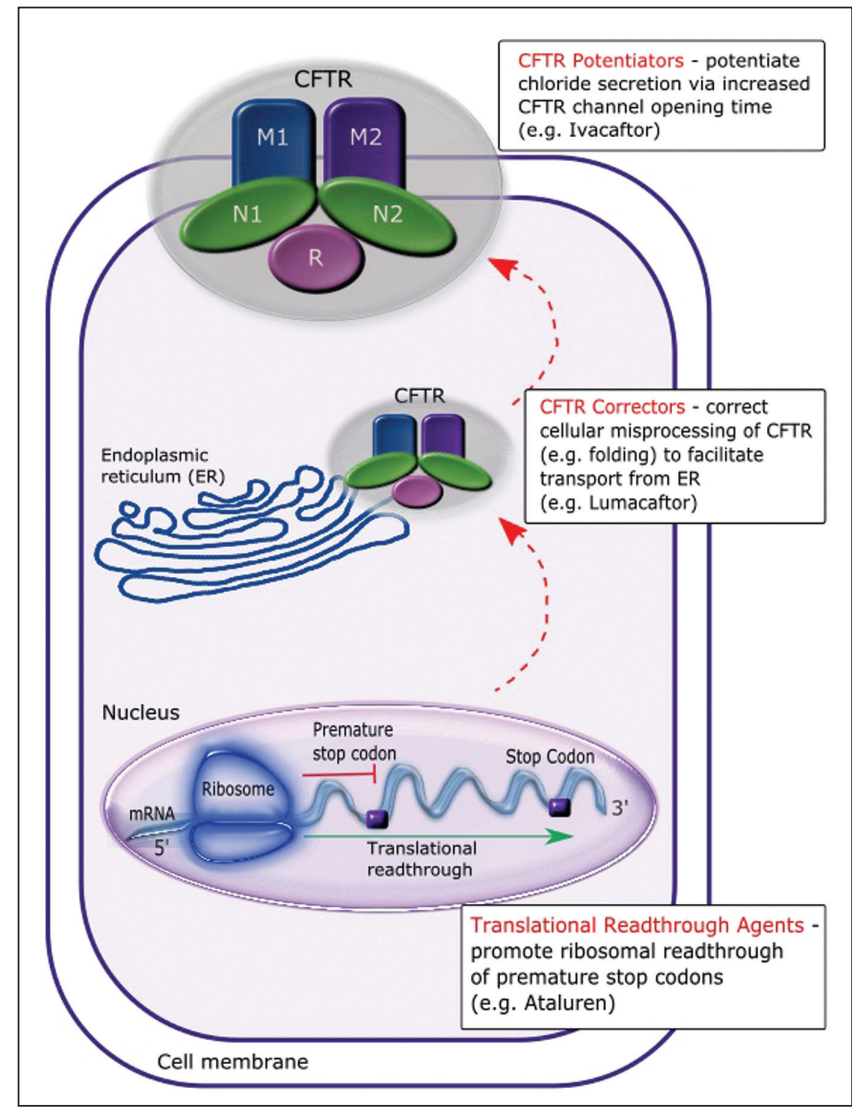

Figure 1) Three targeted sites of action for oral cystic fibrosis transmembrane regulator (CFTR) modulators already approved or in late-phase clinical development for the treatment of cystic fibrosis. ER Endoplasmic reticulum; M1/M2 Membrane-spanning domains of CFTR; mRNA Messenger RNA; N1/N2 Nucleotide-binding domains of CFTR; R Regulatory domain of CFTR

${ }^{1}$ Centre for Heart Lung Innovation, University of British Columbia and St Paul's Hospital; ${ }^{2}$ Division of Respiratory Medicine, Department of Medicine, University of British Columbia, Vancouver, British Columbia

Correspondence: Dr Bradley S Quon, Division of Respiratory Medicine, Department of Medicine, University of British Columbia,

1081 Burrard Street, 8B Providence Wing, Vancouver, British Columbia V6Z 1Y6. Telephone 604-787-3536, e-mail bradley.quon@hli.ubc.ca 
TABLE 1

Cystic fibrosis transmembrane regulator (CFTR) mutational classes and impact on CFTR structure and function

\begin{tabular}{|c|c|c|c|c|c|c|}
\hline & \multicolumn{6}{|c|}{ Mutational class } \\
\hline & $\mathbf{I}$ & II & III & IV & $\mathbf{V}^{*}$ & VI \\
\hline Type of mutation & $\begin{array}{l}\text { Nonsense } \\
\text { Frameshift } \\
\text { Splice }\end{array}$ & $\begin{array}{l}\text { Missense } \\
\text { AA deletion }\end{array}$ & $\begin{array}{l}\text { Missense } \\
\text { AA change }\end{array}$ & $\begin{array}{l}\text { Missense } \\
\text { AA change }\end{array}$ & $\begin{array}{l}\text { Missense } \\
\text { Splice }\end{array}$ & $\begin{array}{l}\text { Missense } \\
\text { AA change }\end{array}$ \\
\hline $\begin{array}{l}\text { CFTR genotypes } \\
\text { with }>1 \% \text { prevalence } \\
\text { in Canada }\end{array}$ & $\begin{array}{c}621+1 \mathrm{G} \rightarrow \mathrm{T}(6 \%) \\
\mathrm{G} 542 \mathrm{X}(4 \%) \\
\mathrm{W} 1282 \mathrm{X} \\
\mathrm{R} 553 \mathrm{X} \\
\mathrm{R} 1662 \mathrm{X} \\
2183 \mathrm{AA} \rightarrow \mathrm{G}\end{array}$ & $\begin{array}{l}\text { F508del }(90 \%) \\
\text { N1303K }\end{array}$ & G551D (3\%) & $\mathrm{R} 117 \mathrm{H}(2 \%)$ & $\begin{array}{c}\mathrm{A} 455 \mathrm{E}(3 \%) \\
3849+10 \mathrm{~kb} \mathrm{C} \rightarrow \mathrm{T} \\
2789+5 \mathrm{G} \rightarrow \mathrm{A} \\
3120+1 \mathrm{G} \rightarrow \mathrm{A} \\
711+1 \mathrm{G} \rightarrow \mathrm{T}\end{array}$ & None \\
\hline CFTR defect & $\begin{array}{l}\text { No functional } \\
\text { CFTR protein }\end{array}$ & $\begin{array}{c}\text { CFTR 'trafficking' } \\
\text { defect }\end{array}$ & $\begin{array}{l}\text { CFTR channel regulation } \\
\text { or 'gating' defect }\end{array}$ & $\begin{array}{c}\text { Decreased CFTR } \\
\text { channel conductance }\end{array}$ & $\begin{array}{l}\text { Reduced CFTR } \\
\text { protein synthesis }\end{array}$ & $\begin{array}{l}\text { Decreased CFTR } \\
\text { membrane stability }\end{array}$ \\
\hline
\end{tabular}

*In an alternative CFTR mutation classification scheme, class V is grouped with class I and class VI becomes class V. AA Amino acid; del Deletion

defective opening or 'gating' of the CFTR chloride channel. CFTR potentiators are small molecules that increase chloride secretion by increasing CFTR channel opening time (Figure 1). Class IV mutations are very similar to class III mutations, but lead to decreased chloride secretion due to reduced channel conductance as opposed to defective channel 'gating'. Class V mutations involve noncoding regions (introns) that affect transcriptional regulation or splicing (ie, the process of preparing RNA for translation) resulting in reduced synthesis of CFTR. Finally, class VI mutations are missense mutations that cause amino acid substitutions that decrease the stability of the CFTR protein at the cell surface, resulting in increased turnover and reduced levels.

\section{CFTR MUTATION-SPECIFIC THERAPIES: THE EVIDENCE}

The landscape of CF therapeutics changed dramatically with the recent approval of ivacaftor (Kalydeco, Vertex Pharmaceuticals Inc, USA), a small-molecule oral CFTR potentiator therapy that targets the defective CFTR protein in patients with a class III G551D mutation. This mutation-specific treatment provided proof-of-concept that personalized therapy in CF was an achievable goal. For the first time, a treatment was available with the potential to ameliorate downstream disease processes resulting from CFTR dysfunction, namely mucus, infection and inflammation. In this section, we review the clinical trial evidence supporting ivacaftor and other modulators of CFTR currently in the therapeutic pipeline. A PubMed search from January 1, 2008 to January 1, 2015 was conducted and included the search terms "cystic fibrosis" and "clinical trials". Initially, 100 articles were screened; however, the search was narrowed to therapies in late-phase clinical development targeting the basic CFTR defect.

\section{Targeting class III mutations}

G551D is the most common class III CFTR 'gating' mutation, with an overall prevalence of $3 \%$ in Canada (5). More than 228,000 small molecules were initially screened to identify 'potentiators' of CFTR gating (6). VX-770, later known as ivacaftor, increased CFTR-mediated chloride transport up to $50 \%$ of wild-type levels in cell culture systems derived from patients with a G551D mutation. It was evaluated in a pivotal phase III, 48-week placebo-controlled study involving patients $\geq 12$ years of age with at least one copy of the G551D mutation (7). Within two weeks of initiation, mean sweat chloride (a biomarker of CFTR function) levels decreased by approximately $50 \%$ to a level below the typical diagnostic threshold for CF compared with no change for the placebo group, and forced expiratory volume in $1 \mathrm{~s}\left(\mathrm{FEV}_{1}\right)$ percent predicted improved by an absolute of $10 \%$ compared with placebo. Following 48 weeks of treatment, the improvement in lung function was maintained and subjects on ivacaftor experienced a mean weight gain of $3 \mathrm{~kg}$, improved respiratory symptoms and a $55 \%$ reduction in the rate of pulmonary exacerbations compared with the placebo group. These treatment effects have been reproduced in patients six to 11 years of age with a G551D mutation (8). Based on the results of these two phase III clinical trials, ivacaftor was well tolerated and the incidence of adverse events was similar to placebo groups $(7,8)$.

Ivacaftor has subsequently been evaluated and approved for use in patients $\geq 6$ years of age, with nine other gating mutations (G178R, S549N, S549R, G551S, G970R, G1244E, S1251N, S1255P and G1349D) (9). Collectively, these mutations account for only approximately $1 \%$ of CF patients. A study is ready to begin evaluating the use of ivacaftor in children two through five years of age with gating mutations in at least one allele.

\section{Targeting class II mutations}

The impact of personalized medicine will be augmented when robust treatments are available for patients with the delta F508 mutation. At least one copy of this class II mutation is encountered in $90 \%$ of Canadians with CF and 50\% are homozygous (10). This deletion mutation is challenging as a therapeutic target because it results in a misfolded protein that is trapped and prematurely destroyed in the endoplasmic reticulum. In addition to impairing CFTR trafficking, it results in decreased CFTR membrane stability and diminished function. This latter functional deficit provided the rationale to trial ivacaftor in patients homozygous for the delta F508 mutation. Based on the results of a phase II study involving 140 patients over a 16-week period, ivacaftor resulted in a small improvement in sweat chloride level compared with placebo; however, this did not translate into clinically significant improvements (11).

Subsequent efforts have focused on combining ivacaftor with CFTR correctors capable of increasing the processing and delivery of CFTR to the cell surface. Using an approach similar to the discovery of ivacaftor, 164,000 small molecules were screened in cell-based assays and VX-809, later known as lumacaftor, was identified as a lead compound (12). Lumacaftor increased CFTR-mediated chloride transport to $14 \%$ of wild-type levels. While having a less dramatic effect on CFTR function compared with ivacaftor in G551D, this small molecule was advanced to clinical development because data suggested that rescue of CFTR function to $10 \%$ of wild-type levels could reduce disease severity (13). Based on the results of a phase II study involving CF adults homozygous for the delta F508 mutation, lumacaftor alone had negligible effect on lung function but when combined with ivacaftor resulted in a modest $4 \%$ to $8 \%$ absolute improvement in $\mathrm{FEV}_{1}$ (14). Two near-identical phase III studies evaluating lumacaftor and ivacaftor in 1000 patients homozygous for the delta F508 mutation and $\geq 12$ years of age has just been completed (15). Both studies met their primary end points, with a $3 \%$ to $4 \%$ absolute increase in $\mathrm{FEV}_{1}$ over a 24-week period compared with placebo. There was also a $30 \%$ to $39 \%$ reduction in the rate of pulmonary exacerbations in the lumacaftor-ivacaftor groups compared with placebo. Based on 
these results, Vertex Pharmaceutical Inc (USA) has submitted a new drug application for approval in the United States and Europe. Vertex Pharmaceuticals Inc has another promising CFTR corrector known as VX-661 in its pipeline, which will also be evaluated in combination with ivacaftor in four phase III studies set to launch sometime in 2015.

\section{Targeting class I mutations}

Aminoglycosides are capable of promoting ribosomal translational readthrough of premature stop codons (16); however, the high concentrations required to achieve this therapeutic effect can lead to oto- and nephrotoxicity. An orally administered small molecule translational readthrough agent, PTC124 (later known as Ataluren, PTC Therapeutics Inc, USA), was discovered using high-throughput screening approaches (17). Safety was demonstrated in early phase clinical studies and it was recently evaluated in a phase III multicentre placebo-controlled study involving 238 patients (age $\geq 6$ years) with a nonsense mutation (18). This study did not meet its primary end point because there was no improvement in lung function over a 48-week period, and there was a signal for increased risk for acute kidney injury ( $15 \%$ versus $<1 \%)$ compared with placebo. In a post hoc analysis of patients not receiving chronic inhaled tobramycin, there was a statistically significant improvement in $\mathrm{FEV}_{1}$ and a reduction in the rate of exacerbations but the effects were modest. A phase III study has just started evaluating ataluren in patients with a nonsense mutation but not on chronic inhaled aminoglycosides.

\section{Targeting class IV to VI mutations}

$\mathrm{R} 117 \mathrm{H}$ is a class IV mutation with a prevalence of approximately $2 \%$ in Canada. By definition, this mutation leads to reduced CFTR channel conductance, but it also has properties consistent with class III gating mutations. Ivacaftor was evaluated in 70 patients $\geq 6$ years of age with at least one R117H mutation (Table 2). While the results have not yet been published, the study did not meet its primary end point in terms of $\mathrm{FEV}_{1}$ improvement for all subjects included, but there was a significant improvement in $\mathrm{FEV}_{1}$ in a prespecified exploratory subgroup analysis of adults enrolled in the study. As a result, Health Canada recently approved this therapy for patients $\geq 18$ years of age with at least one $\mathrm{R} 117 \mathrm{H}$ mutation. Our Pubmed search did not identify any agents in late phase clinical development targeting class V or VI mutations; however, there are gene-based therapies in the pipeline.

\section{FUTURE RESEARCH AND CHALLENGES IN THE ROAD AHEAD}

The CF community eagerly awaits the results of a clinical trial evaluating gene replacement therapy using a cationic lipid carrier vector (2). If this is efficacious, a single therapy may be available to treat all mutational classes. For existing CFTR modulators, the longest clinical trial conducted to date has been 48 weeks in duration; therefore, long-term studies are needed to evaluate effectiveness and safety in what may be a lifelong therapy. Because these molecules act on the underlying defect, it would appear logical to start CFTR modulators early after birth; however, studies to support safety in newborns/infants have not yet been conducted. While combined CFTR potentiator and corrector therapy in patients homozygous for the delta F508 mutation appears promising, the clinical improvement is modest. A drug cocktail of multiple CFTR potentiators and correctors may be required to restore robust CFTR function.

While not specific to CF, one of the most significant challenges with personalized medicine moving forward will be the financial burden to the health care system. Ivacaftor costs approximately $\$ 306,000$ per year and, therefore, the budgetary impact of ivacaftor for individuals with a single G551D mutation across Canada (assuming $80 \%$ are eligible) is estimated at $\$ 29$ million. With new mutationspecific therapies on the horizon in CF, cost-effectiveness studies are warranted to assist with provincial funding decisions because some of

\section{TABLE 2}

\section{List of recommendations for application of cystic fibrosis transmembrane regulator (CFTR) modulators in clinical practice and for future research}

- Ivacaftor (Kalydeco, Vertex Pharmaceuticals Inc, USA) is a first-in-class orally administered therapy that acts on the underlying cystic fibrosis defect by potentiating activity of the CFTR protein

- Ivacaftor is well-tolerated, safe and efficacious in patients with class III gating mutations ( $3 \%$ of Canadians with cystic fibrosis)

- Combinations of CFTR potentiators and correctors are necessary to restore CFTR function in patients with class II deltaF508-CFTR mutations

- Ataluren (PTC Therapeutics Inc, USA) promotes translational readthrough and may benefit patients with class I nonsense mutations not on chronic inhaled aminoglycosides

- Long-term studies are required to evaluate the impact of CFTR modulators on modifying disease course, especially if started early in life

the up-front medication-related costs may be offset by reduced downstream costs and burden related to the disease.

\section{CONCLUSIONS}

$\mathrm{CF}$ can serve as a potential paradigm for the application of personalized medicine in other respiratory diseases and genetic conditions. The breakthroughs achieved to date offer tremendous hope to many patients affected by this life-shortening condition. Although further research is required to restore robust CFTR function in all individuals with CF, there are many reasons to believe this remains a realistic goal.

FUNDING: BSQ receives salary support from Cystic Fibrosis Canada and the University of British Columbia.

DISCLOSURES: The authors have no additional financial disclosures or conflicts of interest to declare.

\section{REFERENCES}

1. Kerem B, Rommens JM, Buchanan JA, et al. Identification of the cystic fibrosis gene: Genetic analysis. Science 1989;245:1073-80.

2. Armstrong DK, Cunningham S, Davies JC, Alton EW. Gene therapy in cystic fibrosis. Arch Dis Childhood 2014;99:465-8.

3. CFTR2. Clinical and Functional Translation of CFTR. Baltimore: CF Foundation, John Hopkins University, The Hospital for Sick Children. <www.cftr2.org/> (Accessed April 2, 2015).

4. Sosnay PR, Siklosi KR, Van Goor F, et al. Defining the disease liability of variants in the cystic fibrosis transmembrane conductance regulator gene. Nat Genetics 2013;45:1160-7.

5. Boyle MP, De Boeck K. A new era in the treatment of cystic fibrosis: Correction of the underlying CFTR defect. Lancet Respir Med 2013;1:158-63.

6. Van Goor F, Hadida S, Grootenhuis PD, et al. Rescue of CF airway epithelial cell function in vitro by a CFTR potentiator, VX-770. Proc Natl Acad Sci U S A 2009;106:18825-30.

7. Ramsey BW, Davies J, McElvaney NG, et al. A CFTR potentiator in patients with cystic fibrosis and the G551D mutation. N Engl J Med 2011;365:1663-72.

8. Davies JC, Wainwright CE, Canny GJ, et al. Efficacy and safety of ivacaftor in patients aged 6 to 11 years with cystic fibrosis with a G551D mutation. Am J Respir Crit Care Med 2013;187:1219-25.

9. De Boeck K, Munck A, Walker S, et al. Efficacy and safety of ivacaftor in patients with cystic fibrosis and a non-G551D gating mutation. J Cystic Fibrosis 2014;13:674-80.

10. Cystic Fibrosis Canada. The Canadian Cystic Fibrosis Registry. 2013 Annual Report. <www.cysticfibrosis.ca/cf-care/cf-registry/> (Accessed February 12, 2015).

11. Flume PA, Liou TG, Borowitz DS, et al. Ivacaftor in subjects with cystic fibrosis who are homozygous for the F508del-CFTR mutation. Chest 2012;142:718-24. 


\section{Quon and Wilcox}

12. Van Goor F, Hadida S, Grootenhuis PD, et al. Correction of the F508del-CFTR protein processing defect in vitro by the investigational drug VX-809. Proc Natl Acad Sci U S A 2011;108:18843-8.

13. Rowe SM, Accurso F, Clancy JP. Detection of cystic fibrosis transmembrane conductance regulator activity in early-phase clinical trials. Proc Am Thorac Society 2007;4:387-98.

14. Boyle MP, Bell SC, Konstan MW, et al. A CFTR corrector (lumacaftor) and a CFTR potentiator (ivacaftor) for treatment of patients with cystic fibrosis who have a phe508del CFTR mutation: A phase 2 randomised controlled trial. Lancet Respir Med 2014;2:527-38.

15. Wainright CE, Elborn JS, Ramsey BW, et al. Lumacaftor-ivacaftor in patients with cystic fibrosis homozygous for Phe508del CFTR. N Engl J Med. May 17, 2015 (Epub ahead of print).
16. Wilschanski M, Yahav Y, Yaacov Y, et al. Gentamicin-induced correction of CFTR function in patients with cystic fibrosis and CFTR stop mutations. N Engl J Med 2003;349:1433-41.

17. Welch EM, Barton ER, Zhuo J, et al. PTC124 targets genetic disorders caused by nonsense mutations. Nature 2007;447:87-91.

18. Kerem E, Konstan MW, De Boeck K, et al. Ataluren for the treatment of nonsense-mutation cystic fibrosis: A randomised, double-blind, placebo-controlled phase 3 trial. Lancet Respir Med 2014;2:539-47. 


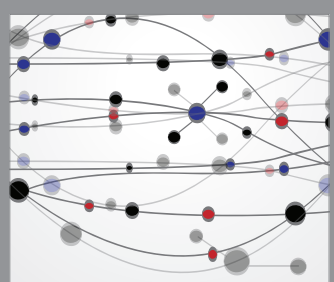

The Scientific World Journal
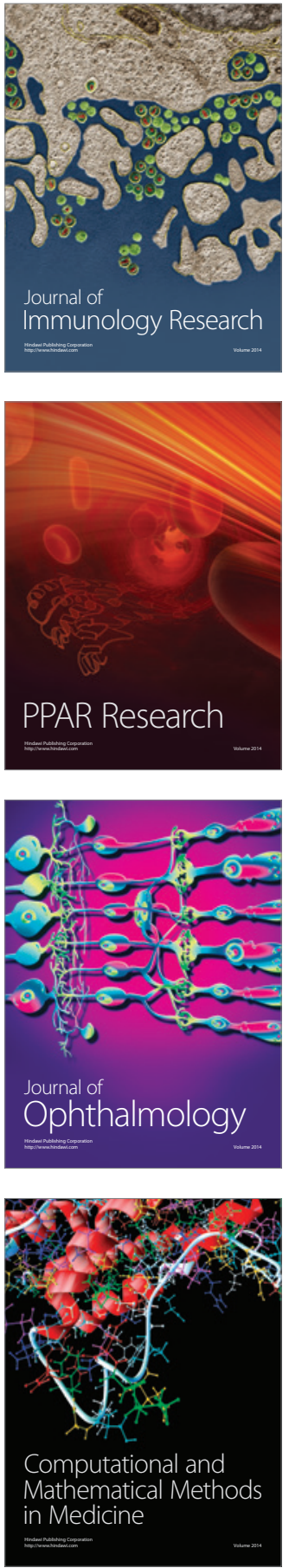

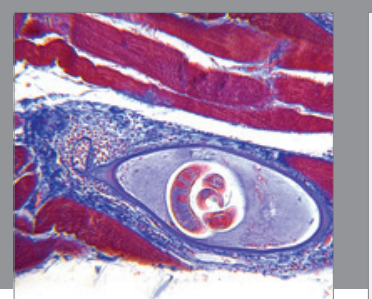

Gastroenterology Research and Practice

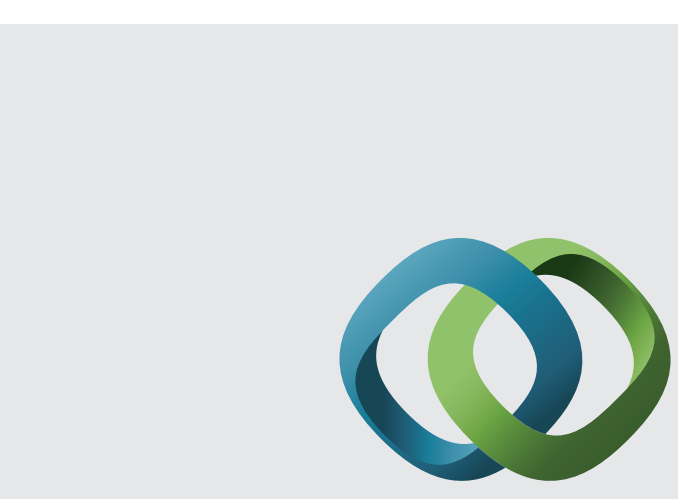

\section{Hindawi}

Submit your manuscripts at

http://www.hindawi.com
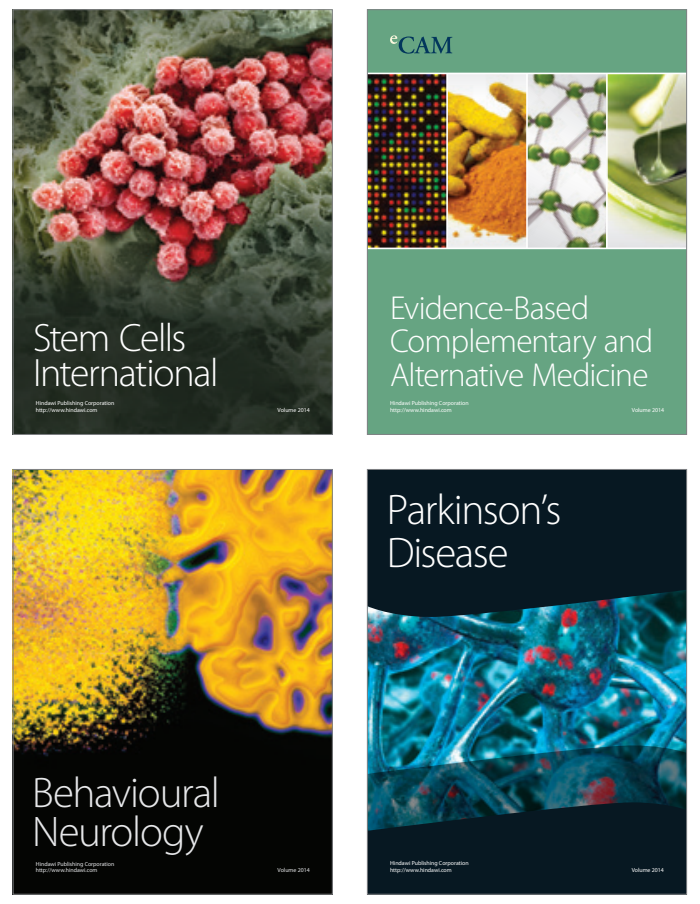
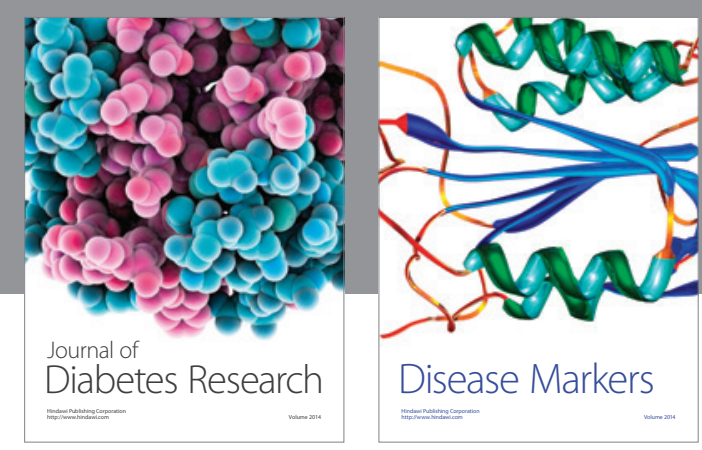

Disease Markers
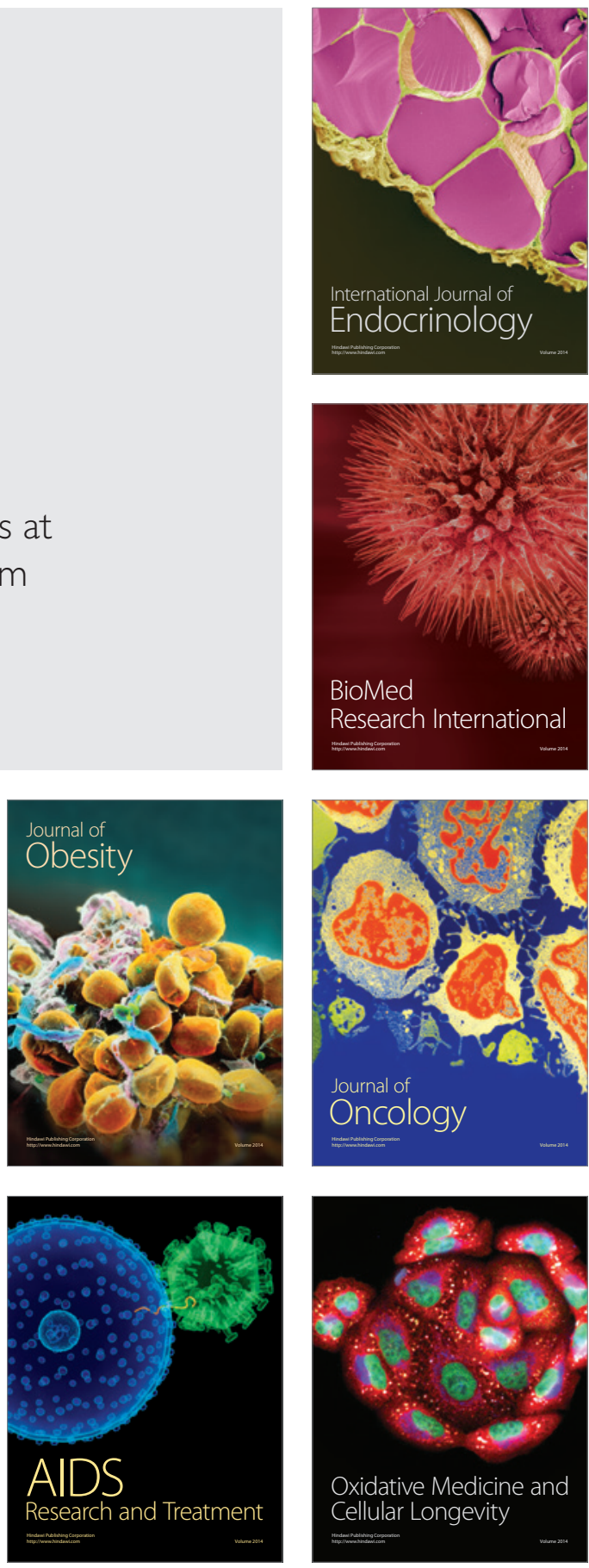